\title{
Magnetic towers and binary-formed disks: New results for planetary nebula evolution
}

\author{
Martín Huarte-Espinosa and Adam Frank \\ Department of Physics and Astronomy, University of Rochester, \\ 600 Wilson Boulevard, Rochester, NY, 14627-0171 \\ emails: martinhe; afrank; @pas.rochester.edu
}

\begin{abstract}
We present new results of 3-D AMR MHD simulations focusing on two distinct aspects of PPN evolution. We first report new simulations of collimated outflows driven entirely by magnetic fields. These Poynting flux dominated "magnetic towers" hold promise for explaining key properties of PPN flows. Our simulations address magnetic tower evolution and stability. We also present results of a campaign of simulations to explore the development of accretion disks formed via wind capture. Our result focus on the limits of disk formation and the range of disk properties.
\end{abstract}

Keywords. planetary nebulae: general, methods: numerical, ISM: jets and outflows, MHD, binaries: general, accretion, accretion disks

\section{Introduction}

Jets are observed in proto-Planetary Nebuale (PPN), Young Stellar Objects, radio galaxies and other astrophysical objects. Models suggest that jets are launched and collimated by accretion, rotation and magnetic mechanisms (Pudritz et al. 2007). One paradigm to explain the formation of jets in PPN is that of a binary system in which material from an AGB star is accreted onto an intermediate-mass companion (Soker \& Rappaport 2000; Nordhaus, Blackman \& Frank 2007). Jets or collimated outflow have recently been observed in the close binaries NGC 6326 and NGC 6778 (Miszalski et al. 2011). Previous numerical studies of wind capture in binaries have found enhancements over Bondi-Hoyle accretion rates onto the secondary (Mastrodemos \& Morris 1998; Podsiadlowski \& Mohamed 2007; De Val-Borro et al. 2009). These results, however, must be tested using high resolution simulations because the implications for the maximum outflow power are dramatic; the answer could rule in, or out, the secondary as the engine powering jets in PPN.

In the case of magnetized jets, plots of specific angular momentum vs. jet poloidal speed are distinct for different MHD engines (Ferreira et al. 2006). This fact can help constrain the physics of the jet engine. The importance of the magnetic fields relative to the flows' kinetic energy, divides jets into (i) Poynting flux dominated (PFD; Shibata \& Uchida 1986), in which magnetic fields dominate the jet structure, (ii) magnetocentrifugal (Blandford \& Payne 1982), in which magnetic fields only dominate out to the Alfvén radius. The observable differences between PFD and magnetocentrifugal jets are unclear, as are the effects of cooling and rotation on PFD jets. Recently, cooling magnetized jets have been formed in laboratory experiments (Lebedev et al. 2005). Such studies, along with high resolution 3-D MHD numerical simulations, have proven to play a key role in the understanding of the physics of jet launch and stability. 


\section{Magnetic towers}

\subsection{Model}

We use the Adaptive Mesh Refinement (AMR) code AstroBEAR2.0 (Cunningham et al. 2009; Carroll-Nellenback et al. 2011) to solve the equations of radiative-MHD in 3D. The grid represents $160 \times 160 \times 400$ AU divided into $64 \times 64 \times 80$ cells plus 2 AMR levels. Initially, the molecular gas is static and has an ideal gas equation of state $(\gamma=5 / 3)$, a number density of $100 \mathrm{~cm} \mathrm{~s}^{-1}$ and a temperature of $10000 \mathrm{~K}$. The magnetic field is helical, centrally localized and given by the vector potential (in cylindrical coordinates) $\mathbf{A}(r, z)=[(r / 4)(\cos (2 r)+1)(\cos (2 z)+1)] \hat{\phi}+[5(\cos (2 r)+1)(\cos (2 z)+1)] \hat{k}$, for $r, z<$ $30 \mathrm{AU}$, and $\mathbf{A}(r, z)=0$ elsewhere. The magnetic pressure is higher than the thermal one inside the magnetized region.

Source terms are used to continually inject magnetic or kinetic energy at cells $r, z<$ 30 AU. We carry out 4 simulations: an adiabatic, a cooling (Dalgarno \& McCray 1972) and a rotating (Keplerian) PDF jet, as well as a hydrodynamical jet which has the same time average propagation speed and energy flux than the adiabatic PFD jet.

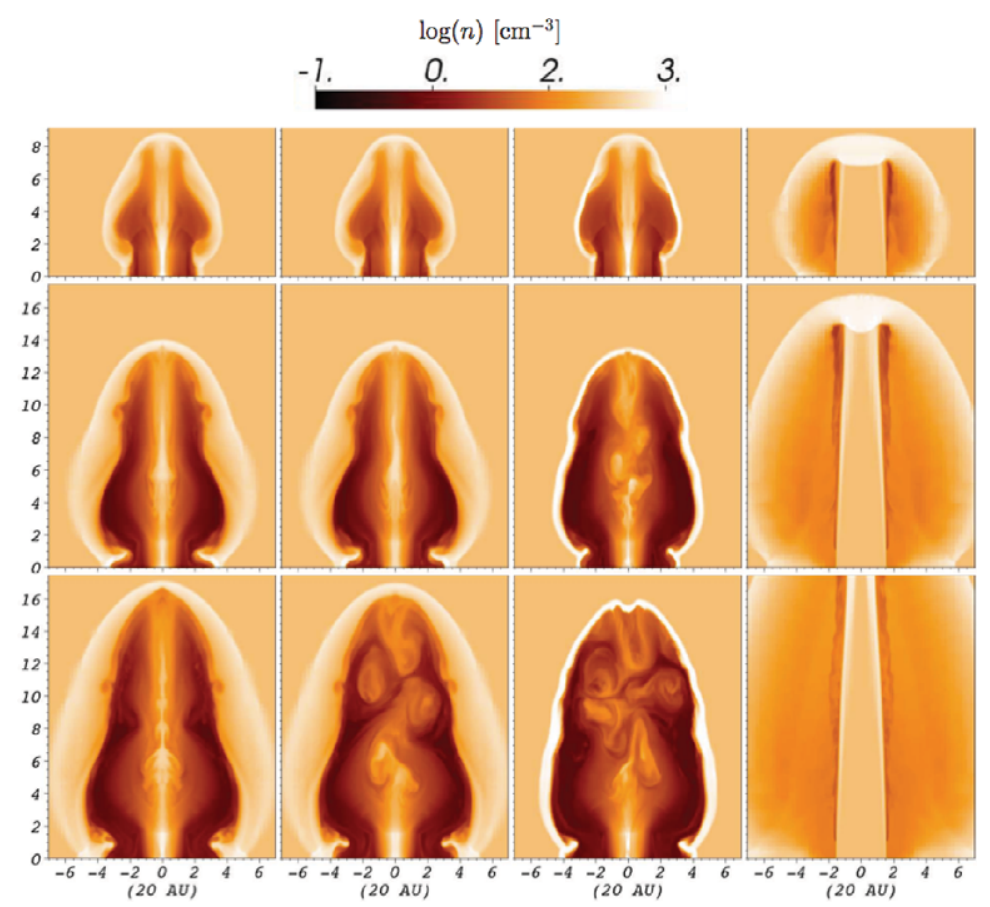

Figure 1. Logarithmic false color number density $\left(\mathrm{cm}^{-3}\right)$ maps of the gas at the midplane of the cubic computational domain. Adiabatic (1st column), rotating (2nd column) and cooling (3rd column) PDF jets. The hydrodynamic jet is in the 4th column. From top to bottom the time is 42,84 and $118 \mathrm{yr}$.

\subsection{Results}

Magnetic pressure pushes field lines and plasma up, forming magnetic cavities with low density. The adiabatic case is the most stable. PFD jets decelerate relative to the hydrodynamic one; magnetic energy pressure produces axial but also radial expansion. PFD jet cores are thin and unstable, whereas the hydrodynamic jet beam is thicker, smoother and stable. The PFD jets are sub-Alfvénic. Their cores are confined by magnetic hoop 


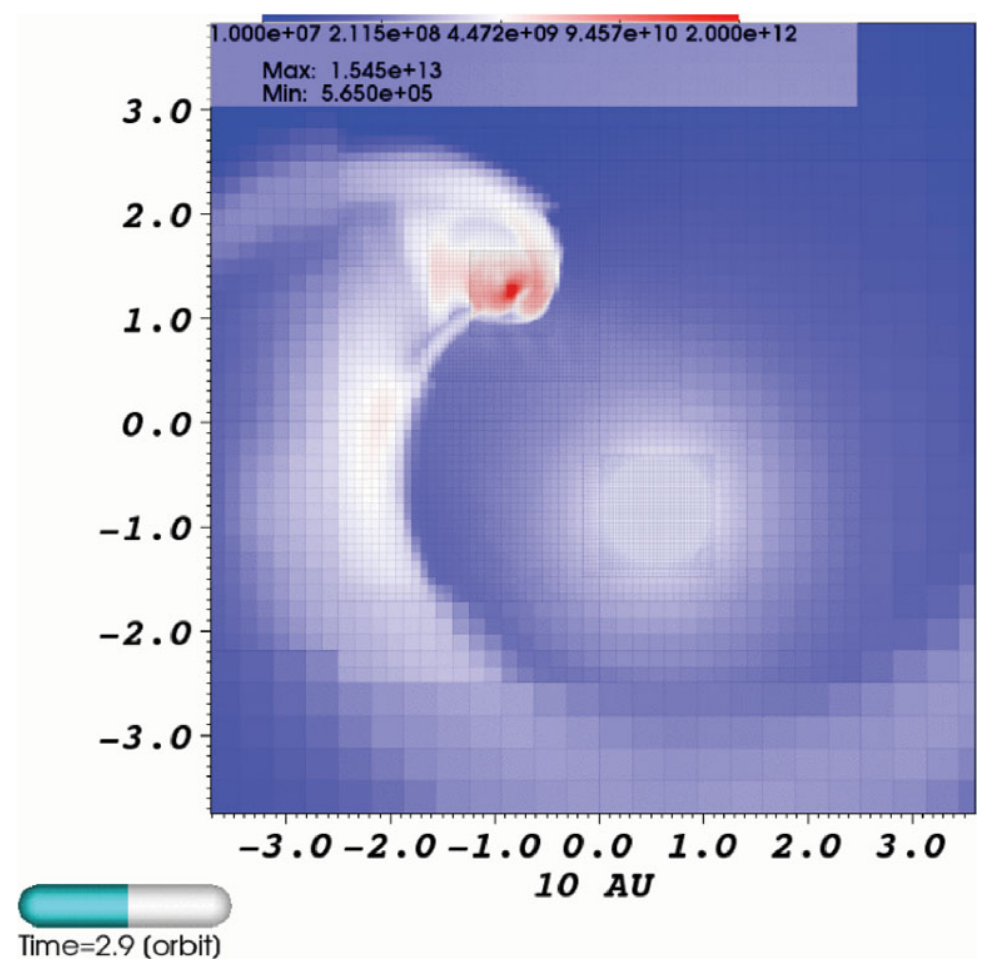

Figure 2. Logarithmic number density $\left(\mathrm{cm}^{-3}\right)$ map of the gas in the orbital plane. The primary and secondary stars are located at $(0.6,-0.9)$ and $(-0.9,1.2)$, respectively. The blue and white components are the AGB wind, the spiral structure of which is caused by the orbital motion. The red gas component is the accretion disk that is formed around the secondary via wind capture.

stress, while their surrounding cavities are collimated by external thermal pressure. PDF jets carry high axial currents which return along their outer contact discontinuity. Pinch, $m=0$, kink, $m=1$, and $m=2$ current-driven perturbations are evident in the PDF jets. The perturbations are amplified by cooling, firstly, and base rotation, secondly. This happens because both shocks and thermal pressure support are weakened by cooling, and the total pressure balance at the jets' base is affected by rotation. See Huarte-Espinosa et al. 2012 (in prep) for details.

\section{Binary-formed disks}

\subsection{Model}

We use Astrobear2.0 (Cunningham et al. 2009; Carroll-Nellenback et al. 2011) to solve the equations of hydrodynamics in $3 \mathrm{D}$. The grid represents $100 \mathrm{AU}^{3}$ divided into $32^{3}$ cells. We also use 5 AMR levels which allow us to have high resolution, of order $0.4 \mathrm{AU}$, around the model stars. These, are implemented with gravity particles, separated by $25 \mathrm{AU}$ and follow circular orbits. The primary simulates an AGB star with a mass of $1.5 \mathrm{M}_{\odot}$, and a spherical constant wind of $10 \mathrm{~km} \mathrm{~s}^{-1}$ and $\dot{M} \sim 10^{-8} \mathrm{M}_{\odot} \mathrm{yr}^{-1}$. The secondary star simulates a main sequence star or a white dwarf with $1 \mathrm{M}_{\odot}$.

Initially, we implement an isothermal $(\gamma=1.001)$ gas in the grid which is unaffected by the gravitational field of either of the stars, but they pull each other around the center of gravity which is located at the origin (Figure 2). The system orbits twice and 
the grid is filled with material from the primary's AGB wind. The orbital motion causes a helical-like distribution on the wind. We then "turn-on" the effect of the secondary's gravity on the gas and allow the system to orbit ten times under these conditions.

\subsection{Results}

As the stars orbit each other, the dense gas from the AGB wind flowing near the secondary is captured by the star's gravitational field. This process is essentially a form of BondiHoyle flow and the flow pattern is localized near the secondary. A spiral shock pattern does however propagate through the AGB wind.

Within one orbital timescale we see that an accretion disk forms around the secondary. We note that previous simulations of accretion disk formation via wind capture in binaries have explored star separations smaller than our model with 25 AU (e.g. Mastrodemos \& Morris 1998). Thus our results show that disks can form even out to large separations.

Preliminary analysis finds the density and temperature of the disk progressively increasing in time. We also find that disk material close to the secondary follows Keplerian orbits confirming it is bound to the star. Finally, our calculations appear to suggest that the disk's total angular momentum vector tilts with respect to the orbital plane as the simulation evolves. See Huarte-Espinosa et al. 2012b (in prep) for details.

\section{Conclusions and Summary}

PFD jet beams are lighter, slower and less stable than kinetic-energy dominated ones. We predict characteristic emission distributions for each of these. Current-driven perturbations in PFD jets are amplified by cooling, firstly, and base rotation, secondly: shocks and thermal pressure support are weakened by cooling. Total pressure balance at the jets' base is affected by rotation. Our simulations are in good agreement with the models and experiments of Shibata \& Uchida 1986 and Lebedev et al. 2005, respectively.

Regarding the problem of the accretion disk formed by wind capture, we see the formation of accretion disks at large binary separations of order $25 \mathrm{AU}$. The density and temperature of the disk progressively increases in time. Our calculations seem to suggest that the disk's total angular momentum vector tilts as the simulation evolves.

\section{References}

Blandford, R. D. \& Payne, D. G. 1982, MNRAS, 199, 883

Carroll-Nellenback, J. J., Frank, A., Shroyer, B., \& Ding, C. 2012, (in prep)

Cunningham A. J., Frank, A., Varnière, P., Mitran, S., \& Jones, T. W. 2009, ApJS, 182, 519

Dalgarno A. \& McCray R. A. 1972, ARA\&A, 10, 375

de Val-Borro, M., Karovska, M., \& Sasselov, D. 2009, ApJ, 700, 1148

Ferreira, J., Dougados, C., \& Cabrit, S. 2006, AAP, 453, 785

Huarte-Espinosa, M., Frank, A., Blackman, E. G., Lebedev, S., \& Ciardi, A. 2012, (in prep)

Huarte-Espinosa, M., Carroll-Nellenback, J. J., Frank, A., Blackman, E. G., \& Nordhaus, J. 2012 , (in prep)

Lebedev, S. V., et al. 2005, MNRAS, 361, 97

Mastrodemos, N. \& Morris, M. 1998, ApJ, 497, 303

Miszalski, B., Jones, D., Rodríguez-Gil, P., Boffin, H. M. J., Corradi, R. L. M., \& SantanderGarcía, M. 2011, AAP, 531, A158

Nordhaus, J., Blackman, E. G., \& Frank, A. 2007, MNRAS, 376, 599

Podsiadlowski, P. \& Mohamed, S. 2007, Baltic Astronomy, 16, 26

Pudritz, R. E., Ouyed, R., Fendt, C., \& Brandenburg, A. 2007, Protostars and Planets V, 277

Shibata, K. \& Uchida, Y. 1986, PASJ, 38, 631

Soker, N. \& Rappaport, S. 2000, ApJ, 538, 241 